\title{
Texture perceived with the fingers
}

\author{
E. L. CUSSLER, SUSAN J. ZLOTNICK, and M. C. SHAW \\ Carnegie-Mellon University, Pittsburgh, Pennsylvania 15213
}

\begin{abstract}
Relative assessments of 10 attributes commonly used to describe the texture of liquids on skin were obtained for 14 liquids of widely varying rheological properties. The results were analyzed to show that these judgments can be predicted accurately from the assessments of just three attributes, smoothness, thinness, and warmth. Smoothness was found to be closely related to the forces of contact lubrication, and could be predicted from measurements of coefficients of friction. Thinness was found to be closely related to viscous forces, consistent with other predictions of assessments of thickness and spreadability. Warmth was not studied further. The results show how careful definition of the stimulus on the skin's surface can sharply simplify the interpretation of the psychophysical experiment.
\end{abstract}

This work is in two parts. The objective of the first part was to devise a nonredundant vocabulary to describe texture perceived with the fingers. The following 10 attributes were selected as the initial vocabulary: thick, thin, spreadable, soft, hard, smooth, creamy, dry, warm, and cool. These attributes are the most important descriptors reported for cosmetics (Goldenberg \& de la Rosa, 1971; Prall, 1973; Breuer, Note 1). This vocabulary contains considerable redundancy: For example, if a fluid is assessed as thin, it seems unlikely to be assessed as thick. The list is also uneven because it includes antonyms like warm and cool, but does not include rough, the expected antonym for smooth. Nevertheless, we decided to restrict the vocabulary to the words given because they are those most commonly used, and to delay investigation of the larger group of less cited attributes.

Untrained observers were asked to scale these attributes, using the method of magnitude estimation and fluids of widely varying rheological properties. This wide range of properties was chosen in the expectation that this would effect a wide range of assessments. The observer's assessments were then used in a multiple regression analysis, from which a nonredundant subset of attributes could be selected. It was found that a subset of just three attributes could be used to accurately predict other attributes. This subset thus is a reduced vocabulary, suitable for further study.

The second part of the paper shows how tactile assessments of fluid samples by human subjects can be quantitatively related to the forces acting on the skin's surface. This step, which is outside the

J. E. Moon helped with some of the experiments. This work was partially supported by National Science Foundation Grant GK-32312-A2, and by Petroleum Research Fund Grant PRF6376-AC-7. E.L.C. is supported by National Institutes of Health Research Career Development Award 1K04-Am-70461. usual range of psychophysical investigation, represents a scheme for generalizing empirical correlations like Stevens' law. The nature of this generalization is best suggested by the example of smoothness. Stevens and Harris (1962) showed that for sandpaper

$$
(\text { smoothness }) \propto(\text { grit diameter })^{-1.5} .
$$

This correlation provides a simple means of estimating sandpaper smoothness over the range of grit diameters studied. However, it does not provide a guide for estimating smoothness in other physical situations. It does not predict the smoothness of fluids on the skin or in the mouth. It does not indicate how sandpaper smoothness will be affected by changes in the skin itself. It is a specific result, not a general strategy.

This paper shows that psychophysical correlations like Equation 1 can be generalized by focusing on the physics of the tactile stimulus. This focus implies emphasizing forces acting on the skin, rather than limiting the analysis to the neural processing within the body.

\section{PSYCHOPHYSICAL METHODS}

\section{Stimuli}

The 14 fluids used in these experiments were as follows: A, gel shampoo (Prell, Proctor \& Gamble); B, hand cream (Pacquins, Pfizer); C, hand lotion (Jergens); D, mayonnaise (Hellman's, CPC International); E, liquid shampoo (Clairol); F, L-45 silicone oil, 500 cstks (Union Carbide); G, marshmallow cream (Kraft); $H$, setting gel ("Dippity-Do," Gillette); I, shaving cream ("Foamy," Gillette); J, L-45 silicone oil, 10 cstks (Union Carbide); K, petroleum jelly (Pureline); L, oleic acid (Eastman); M, L-45 silicone oil, 100,000 cstks (Union Carbide); $\mathrm{N}$, shortening ("Crisco," Proctor \& Gamble).

\section{Subjects}

Subjective assessments were made with untrained test panels of 20 to 25 students, who were not paid. 


\section{Procedure}

Each subject was presented with 5-ml samples of all 14 fluids displayed randomly on a glass plate. Each subject was given the samples with written instructions as follows: "During this experiment you will be presented with samples whose (attributes) vary. You will be asked to evaluate the relative (attribute) of these samples. First choose one of the samples as your standard. Touch it and assign to it a number characteristic of its (attribute). Any positive number will do. Then touch a second sample and assign to it a number characteristic of its relative (attribute). For example, if you assigned 10 to your standard and the second sample feels three times more (attribute), assign the number 30 to the second sample. After testing each sample, record your results. Repeat the procedure for each sample, reassessing your standard whenever you want to." Most subjects assessed their standard with one hand and a second fluid with the other hand. Most chose their standard randomly, although a few said that they tried to choose a standard of "average" properties. When a subject had completed his assessments, he was sometimes asked to repeat his assessments for different attributes. However, a subject's experience did not reduce his variance from the average assessments.

Three subjects gave 0 scores for several assessments of softness and dryness; these assessments were eliminated from the analysis. Three other observers apparently inverted their assess. ments of "thick." The fluids given highest scores for "thickness" by these 3 were given the lowest scores by the remaining 19 subjects. Indeed, the reciprocals of the assessments by these 3 were within the range of values given by the others. Therefore, these reciprocals were used in the analysis.

A logarithmic transformation was applied to each assessment, producing a data set symbolized as $w_{i j k}$, where $i, j$, and $k$ identify the subject, the fluid, and the attribute, respectively. This logarith mic transformation was chosen because the distribution of assessments is skewed and because the variance increases with the mean. To correct for the arbitrariness of individual scales, we defined

$$
\mathrm{x}_{\mathrm{ijk}}=\mathrm{w}_{\mathrm{ijk}}-\left[\sum_{\ell=1}^{\mathrm{J}} \mathrm{w}_{\mathrm{i} \ell \mathrm{k}} / \mathrm{J}\right],
$$

where $\mathrm{J}$ represents the total number of fluids, equal to 14 in this case. Because the variations between subjects are not of concern here, we averaged over subjects to obtain

$$
y_{j k}=\sum_{i=1}^{I_{k}} x_{i j k} / I_{k},
$$

where $I_{k}$ is the total number of subjects who assessed attribute $k$.

We now assumed that $y_{1 k}, y_{2 k} \cdots y_{j k}$ are jointly normally distributed. We then sought a subset of size $\mathrm{N}$ such that

$$
y_{j k}=\sum_{l=1}^{N} y_{j \ell} a_{l k}+\varepsilon_{j k} N<j \leqslant K,
$$

where $a_{k}$ are constants to be found by regression, and the $\varepsilon_{j k}$ are errors in this regression. Put in another way, we sought a group of $\mathbf{N}$ attributes that, without loss of generality, can predict the remaining attributes within our expected error. This form of regression is an alternative to factor analysis which has already been used successfully for the texture of foods and fabrics (e.g., Howorth, 1964; Moskowitz \& Kapsalis, 1974).

\section{PSYCHOPHYSICAL RESULTS}

One objective of this paper is to reduce redundancy in the vocabulary used to describe texture as perceived with the fingers. In the more exact terms used above, we want to find a subset of $\mathbf{N}$ attributes from which we can accurately predict the remaining $(K-N)$ attributes. Such a calculation implies a criterion for deciding what constitutes an accurate prediction. As our criterion, we have chosen the familiar $r^{2}$, the average of the square of the coefficient of determination (Goldberger, 1964); we have fixed $\mathrm{N}$ at the point where increases in the subset size did not produce large increases in $\mathrm{r}^{2}$.

On this basis, we decided that this subset should contain 3 attributes. The average $r^{2}$ is 0.44 for 1 attribute, 0.63 for 2 attributes, 0.80 for 3 attributes, and 0.83 for 4 attributes. Additional attributes give less and less improvement: The average $\mathrm{r}^{2}$ predicting the 10th attribute from the remaining 9 is 0.91 .

While choosing the size of this subset seems straightforward, choosing the specific attributes that it contains is much more subjective. There are many subsets that give approximately equivalent results, as shown in Table 1. For example, the three attributes, spreadable, creamy, and warm predict the results only very slightly better than the attributes, thick, smooth, and cool.

These best 10 combinations of the 120 possibilities seem to be permutations of three distinct groups of attributes. The first group contains creamy and smooth; the second group includes spreadable, thick, and thin; and the third group consists of warm and cool. Attributes within groups seem closely correlated, as shown in Figure 1. Attributes from different groups are much less exactly related, as exemplified by Figure 2 . Interestingly, the best 10 pairs of words, also shown in Table 1, almost always in-

\begin{tabular}{|c|c|c|c|c|c|c|}
\hline \multicolumn{3}{|c|}{ The Best 10 Triples } & \multirow{3}{*}{$\begin{array}{c}\text { Average } \mathrm{r}^{2} \\
939 \\
.939\end{array}$} & \multicolumn{2}{|c|}{ The Best 10 Pairs } & \multirow{2}{*}{$\frac{\text { Average } r^{2}}{.802}$} \\
\hline creamy; & spreadable; & warm & & spreadable; & cool & \\
\hline creamy; & thick; & warm & & thin; & cool & .799 \\
\hline creamy; & thin; & warm & .939 & spreadable; & warm & .791 \\
\hline creamy; & spreadable; & cool & .938 & thin; & warm & .788 \\
\hline creamy; & thick; & cool & .937 & creamy; & thin & .782 \\
\hline creamy; & thin; & cool & .937 & creamy; & spreadable & .779 \\
\hline smooth; & thin; & warm & .932 & smooth; & thick & .773 \\
\hline smooth; & thick; & warm & .932 & smooth; & thin & .772 \\
\hline smooth; & thick; & cool & .930 & smooth; & spreadable & .759 \\
\hline smooth; & thin; & cool & .930 & creamy; & dry & .745 \\
\hline
\end{tabular}

Table 1

Subsets of Attributes Which Describe Texture 


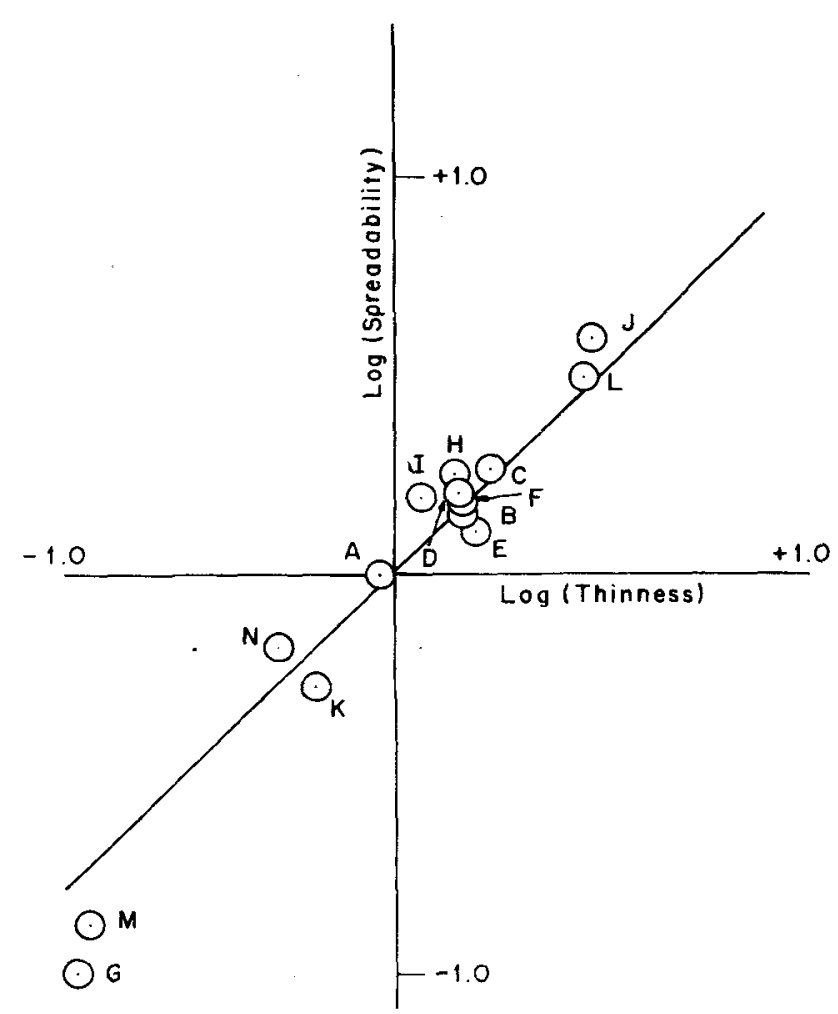

Figure 1. Dependence of spreadability on thinness. These two attributes are apparently from the same group.

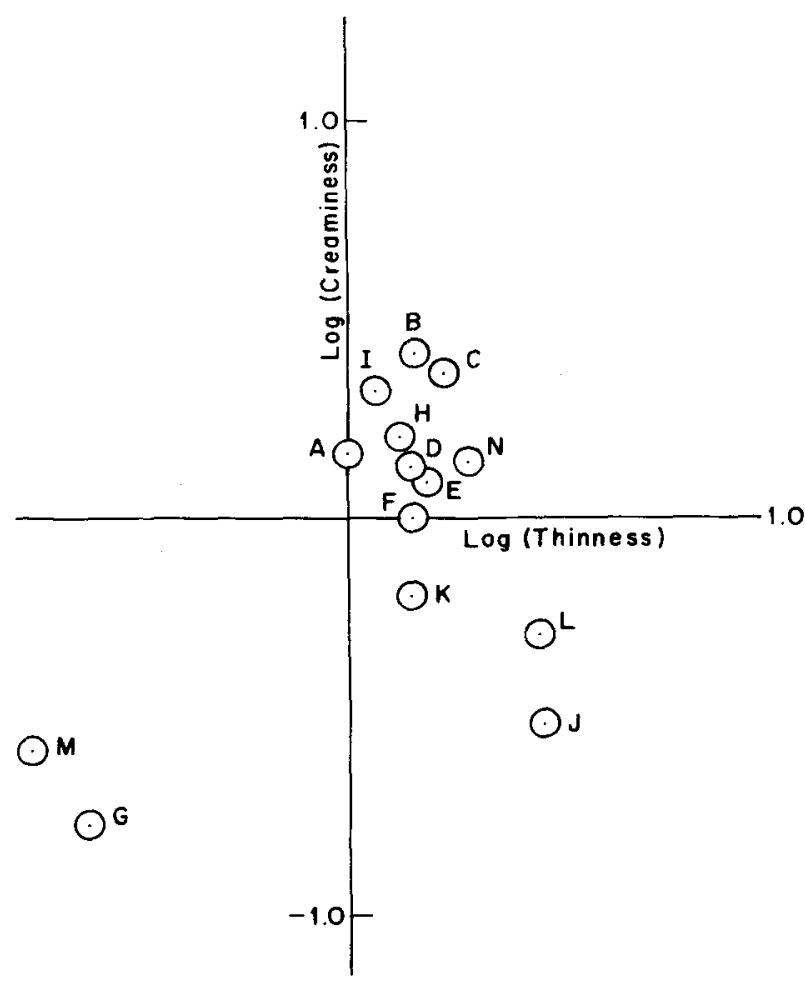

Figure 2. Dependence of creaminess on thinness. These two attributes are from different groups. clude choices from two different groups. We chose one word from each group for further study.

From the first group, we chose smooth instead of creamy, so that we could later compare our results with earlier measurements of the smoothness of sandpaper and of skin (Prall, 1973; Stevens \& Harris, 1962). From the second group, we chose thin, because we wanted to extend earlier measurements of liquid texture (deMartine \& Cussler, 1975). From the third group, we chose warm because it seems to give slightly higher values of $\mathrm{r}^{2}$ than did cool. However, any of the best 10 triples in Table 1 should give similar results.

The regression coefficients found for the three attributes-smooth, thin, warm-are shown in Table 2. The physical implications of these coefficients are more clearly seen by considering the attributes smooth, thin, and warm as three orthogonal axes defining a texture space. Each additional attribute can now be schematically represented in this space as a vector whose coordinates are the coefficients in Table 2 . The projection of these vectors on the smooth-thin plane, shown in Figure 3, gives the dependence of each attribute on smooth and thin. Thus, the coefficients in Table 2 and Figure 3 can be used to calculate the assessment of other attributes from assessments of smoothness, thinness, and warmth. We can also use these coefficients to find several fluids which have one equal attribute but which differ in other attributes.

\section{A THEORY FOR THE TACTILE STIMULUS}

The analysis above showed that assessments of 10 attributes describing texture can be successfully predicted with assessments of just 3 attributes. The analysis below shows how 2 of these 3 attributes, smoothness and thinness, can be correlated with predictable combinations of physical properties.

Such an analysis requires carefully considering the nature of the physical stimulus on the surface of the skin. We assume that the stimulus on the skin is different for the two attributes being considered. For smoothness, we assume that assessments are inversely proportional to the friction force per contact between the fingers. To test this assumption, we idealize each finger as a flat plate moving steadily over a solid surface. The finger is assumed to be in contact with absorbed fluid layers on the surface at a small number of points, consistent with lubrication theory. The frictional force per contact for this motion is (Archard, 1953; Bowden \& Tabor, 1964; Moore, 1975)

$$
\mathrm{F}_{\text {fric }}=\mathrm{sa} \text {, }
$$

where $s$ is the shear strength of the contact and a is 
Table 2

Regression Coefficients Relating Assessments

$\log [$ assessed attribute $k]=a_{1} k \log [$ assessed smoothness $]+a_{2 k} \log [$ assessed thinness $]+a_{3 k} \log [$ assessed warmth]

\begin{tabular}{llrrr}
\hline & $\mathrm{a}_{\mathbf{1 k}}$ & $\mathrm{a}_{\mathbf{2 k}}$ & $\mathrm{a}_{\mathbf{3 k}}$ & $\mathbf{r}^{2}$ \\
\hline Attribute $\mathbf{k}$ & .12 & 1.15 & .04 & .972 \\
spreadable & .79 & .18 & .03 & .878 \\
soft & 1.07 & -1.63 & -.03 & .987 \\
thick & 2.17 & -.86 & -.10 & .918 \\
creamy & .44 & -1.19 & -.23 & .916 \\
hard & .47 & -.79 & .21 & .938 \\
dry & .16 & -.04 & -.84 & .918 \\
\hline
\end{tabular}

the real area of contact. Since the finger is deformable, this area is (Hertz, 1886)

$$
a=\left[\frac{\left(\frac{W}{n_{c}}\right) d}{4 E}-\right]^{2 / 3},
$$

where $E$ is Young's modulus of the finger; $W$ is the total normal load; $n_{c}$ is the total number of contacts; and $d$ is the average diameter of points of contact. Combining these equations yields

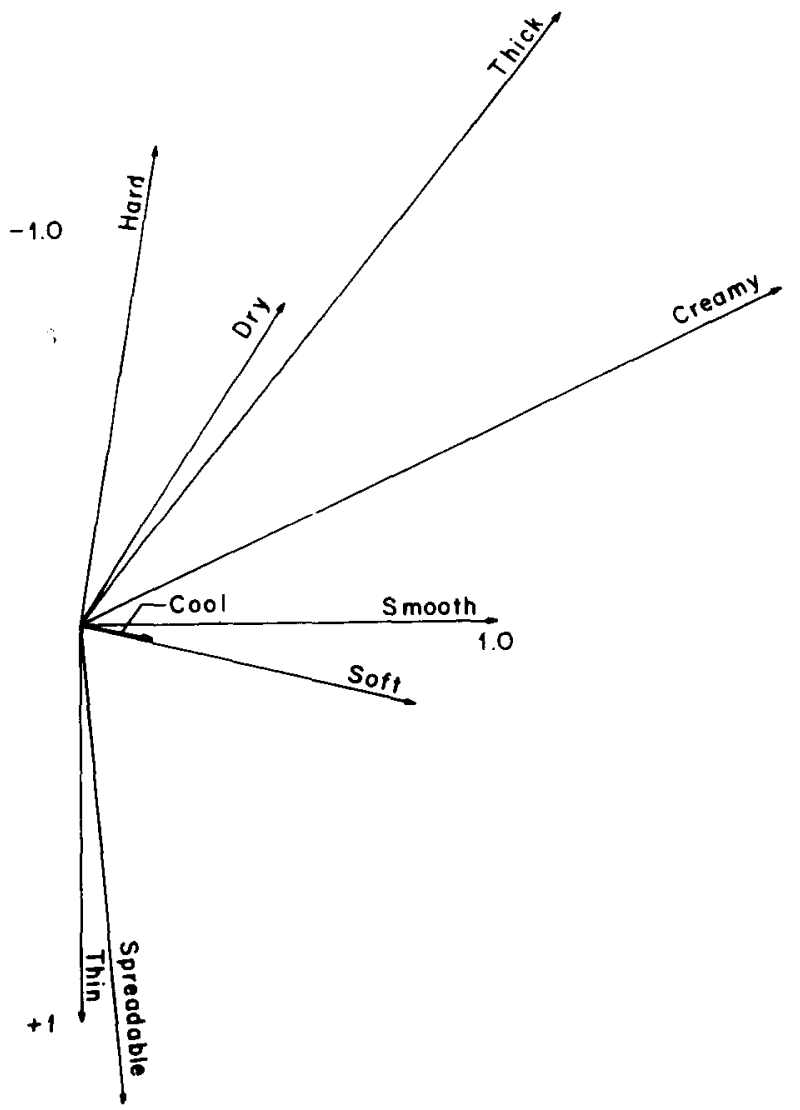

Figure 3. The interdependence of the various attributes. Each attribute can be represented as a vector in a three-dimensional orthogonal space where smooth, thin, and warm represent principal axes. The projection of this space on the smooth-thin plane is shown.

$$
\text { smoothness } \propto \frac{1}{F_{\text {fric }}} \propto \frac{1}{s}\left[\frac{4 E}{\left(\frac{W}{n_{c}}\right) d}\right]^{2 / 3} .
$$

Since all quantities on the right-hand side can be independently measured, Equation 7 can be used to predict relative smoothness.

We want to test this equation for three special cases. First, we want to predict the assessments of the smoothness of liquids, assessments obtained as described above. Here, it is convenient to rearrange Equation 7 as

$$
\text { smoothness } \propto\left\{\frac{1}{\mathrm{~s}}\left[\frac{4 \mathrm{En}_{\mathrm{f}}}{\mathrm{d}}\right]^{2 / 3} \mathrm{~W}^{1 / 3}\right\} \frac{1}{\mathrm{~W}},
$$

where the coefficient of friction, equal to the reciprocal of the quantity in braces, can be measured experimentally. Equation 8, which implies the familiar result that the friction force equals the coefficient of friction times the load (Moore, 1975), will be tested below.

The second special case concerns the smoothness of sandpaper, for which psychophysical data are available (Stevens \& Harris, 1962). In this case, s and $\mathrm{E}$ are constant and $\mathrm{d}$ equals the grit diameter of the sandpaper. As a first approximation, we may assume that the number of grit particles per area is inversely proportional to the area occupied by each particle, so

$$
\mathrm{n}_{\mathrm{c}} \propto \mathrm{d}^{-2} .
$$

Combining Equations 7 and 9 ,

$$
\text { smoothness } \propto \mathrm{s}^{-1} \mathrm{~W}^{-2 / 3} \mathrm{~d}^{-2},
$$

which can also be tested experimentally.

The third special case of Equation 7 is the smoothness of samples of human skin, for which subjective data have also been published (Prall, 1973). The skin samples used were characterized by measurements of scratch resistance and surface topography, defined as the length of the skin's surface divided by the projected length of the same skin sample. We assume that Young's modulus, E, is proportional 
to scratch resistance; that the number of bumps is again inversely proportional to the square of the size; and that bump size is inversely proportional to topography. The first assumption is a common approximation in mechanical engineering, and the second parallels that for sandpaper in Equation 9. The third assumption implies that the topography of a closely folded surface is greater than that for a flat unfolded surface. With these assumptions, Equation 7 can be rewritten as

$$
\begin{gathered}
\text { smoothness } \propto(\text { scratch resistance })^{2 / 3} \\
\cdot(\text { topography })^{2} .
\end{gathered}
$$

This prediction can also be tested experimentally.

We now turn to the assessments of thinness, which is the second attribute being investigated here. We assume that assessments of thinness are inversely proportional to the viscous force per area acting on the skin surface. This viscous force per area is not directly related to the friction force assessed during the perception of smoothness. To calculate this viscous force, we again assume that the fingers act as parallel plates closely separated by a liquid layer. We assume that the liquids behave as power-law fluids (Fredrickson, 1965; Middleman, 1968):

$$
\tau=\mathrm{m}\left(\frac{\partial \mathrm{v}}{\partial z}\right)^{\mathbf{n}},
$$

where $\tau$ is the viscous force per area, $v$ is the velocity of the liquid at a distance $z$ from one finger towards the other, and $m$ and $n$ are experimentally determined rheological parameters. For thin films, this differential equation can be replaced by a difference equation

$$
\boldsymbol{\tau}=\mathbf{m}\left(\frac{\mathrm{v}}{\mathrm{h}}\right)^{\mathrm{n}},
$$

where $\mathrm{V}$ is the velocity of the fingers, and $\mathrm{h}$ is the thickness of the fluid layer on the fingers, a thickness which is itself a function of $m$ and $n$.

Several characteristics of Equation 13 are worth noting. For Newtonian fluids, $n$ equals one and the viscous force per area is proportional to the finger velocity. Such fluids have been studied in previous psychophysical experiments (Stevens \& Guirao, 1964). However, many fluids do not show this simple variation, including everyday materials like most sauces, many soups, and almost all cosmetics. For these non-Newtonian fluids, $\mathrm{n}$ is commonly less than 1 and the viscous force varies less than linearly with the velocity. Both Newtonian and non-Newtonian fluids are of interest here.

The viscous force per area between the fingers can be shown to be (deMartine \& Cussler, 1975): thinness $\propto\{\text { viscous force per area }\}^{-1}$

$$
\propto\left\{m V^{n}\left[\frac{W}{R n+3} \frac{2 \pi m}{n+3}\right]^{\frac{n}{n+1}} \cdot\left[\frac{(n+1) t}{2 n+1}\right]^{\frac{n^{2}}{n+1}}\right\}^{-1},
$$

where $\mathrm{W}$ is the fingers' normal force or load, $\mathrm{R}$ is the effective finger radius, and $t$ is the assessment time. The equation omits a term for the initial thickness of the liquid on the fingers because this term does not significantly affect the calculations (deMartine \& Cussler, 1975). Since all quantities on the righthand side of Equation 14 can be measured experimentally, the equation provides predictions of thinness which will be tested experimentally.

\section{PHYSICAL MEASUREMENTS}

In addition to the subjective assessments given above, we need measurements of the coefficient of friction and of the non-Newtonian viscosity for each of the fluids studied. The coefficient of friction was measured as a function of load with a rotating disk apparatus (Fort, 1962; Moore, 1975). Because we could not obtain reproducible results with available samples of skin, we used samples of chamois. The coefficient of friction was measured at the observed finger velocity as a function of load. The appropriate value for predicting finger perception was then found by interpolation using the measured value of finger load (Kokini, 1976). These cha. nois surfaces did not give the same coefficients of friction as skin, but they did give the same relative values for different types of lubricated surfaces. How major an approximation this represents can only be tested by the results below.

Viscosity was measured in three ways (van Wazer, 1963). For the Newtonian oils, it was determined using Cannon-Ubbelodhe capillary viscometers. For all but the most viscous non-Newtonian fluids, it was measured with a Rheomat 15 cone-and-plate viscometer. For the most viscous materials it was measured with a Sieglaff-McKelvey Instron Model R-64 fitted with a $0.085-\mathrm{cm}$-diam capillary head. Measurements of the 100,000 ctsk oil with all methods gave results within $3 \%$. Several other quantities must be measured to calculate the friction and viscous forces. The normal force or load during assessments was measured by asking subjects to feel sandpaper or to spread fluid samples on the pan of a top-loading Mettler balance and by recording the force. The finger velocity, measured directly, was close to the values found earlier (deMartine \& Cussler, 1975). The fingertips are idealized as circular plates, $1.5 \mathrm{~cm}$ in diameter. Considering the tips as ellipses complicates the equations without improving the predictions. 


\section{COMPARISON OF THEORY WITH PSYCHOPHYSICAL DATA}

The object of this work is to discover combinations of physical parameters which correlate closely with important subjective assessments. This requires idealizing the geometry of the fingers and assuming that each perception is closely related to specific forces on the skin's surface. We have idealized the fingers as two parallel plates steadily sliding over each other. We have also assumed that perceived smoothness is inversely proportional to the frictional force caused by contact between the fingers and a second surface (cf. Equation 7). We want to test this assumption for three separate situations: for fluids, for sandpaper, and for skin.

The predictions of the smoothness of fluids on the skin are summarized in Table 3 . The first three columns identify the fluid, the average load actually used in assessments of its smoothness, and the coefficient of friction at this load. The fourth column gives the actual friction force, found from the first three columns and Equation 8. The friction forces do not vary as widely as the viscous forces calculated below. This is because when we began the experiments we did not know that both coefficient of friction and viscosity would be important. As a result, we inadvertently chose samples for which the coefficients of friction were much more similar than the viscosities. The fifth column in Table 3 reports the assessments of smoothness, found as described above.

The relation between smoothness and friction force is clearer from the results in Figure 4. The data on the ordinate in this figure are the assessments of smoothness: they are purely psychophysical measurements. The data on the abscissa are friction forces: they represent a nonlinear combination of physical factors, as suggested by Equation 8 . The correlation coefficient between the subjective assessments and

Table 3

Physical Parameters Needed to Estimate Smoothness*

\begin{tabular}{ccccc}
\hline Fluid & $\begin{array}{c}\text { Finger } \\
\text { Load }\end{array}$ & $\begin{array}{c}\text { Coefficient } \\
\text { of Friction }\end{array}$ & $\begin{array}{c}\text { Friction } \\
\text { Force }\end{array}$ & Smoothness \\
\hline A & .49 & .17 & .085 & 1.35 \\
B & .29 & .16 & .047 & 1.51 \\
C & .34 & .19 & .064 & 1.64 \\
D & .15 & .72 & .106 & 1.38 \\
E & .34 & .28 & .078 & 1.58 \\
F & .20 & .57 & .112 & 1.18 \\
G & .69 & .68 & .467 & .27 \\
H & .29 & .21 & .061 & 1.39 \\
I & .29 & .28 & .084 & 1.43 \\
J & .20 & .52 & .102 & .92 \\
K & .39 & .30 & .119 & 1.00 \\
L & .34 & .47 & .162 & 1.11 \\
M & .69 & .37 & .252 & .30 \\
N & .34 & .31 & .104 & .72 \\
\hline
\end{tabular}

*Units: finger load, estimated friction force $[=]$ newtons.

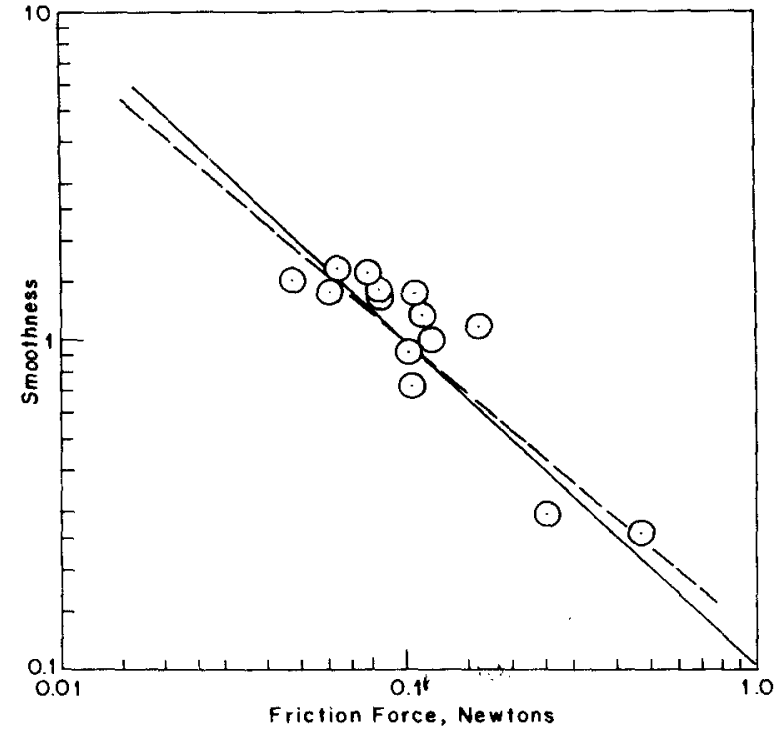

Figure 4. Smoothness vs. friction force. The psychophysical results are shown on the ordinate; the forces on the abscissa are those calculated from the physical measurements in Table 3 . The solid line has slope of -1.0 ; the dotted line of slope -0.88 is the least squares fit.

the friction forces is -0.90 , but this correlation rests strongly on the two outlying points.

We next test the predictions of sandpaper smoothness suggested by Equation 10 . In the physical experiments, we found that the load $W$ varies with the $(-0.3)$ power of the sandpaper's grit diameter. Thus according to Equation 10, smoothness should be proportional to the $(-1.8)$ power of the grit diameter, in reasonable agreement with the $(-1.5)$ power given in Equation 1 (Stevens \& Harris, 1962). The correlation coefficient of the predictions of Equation 10 and Stevens' actual data is 0.97 . One reviewer correctly suggested that Stevens' data are better fit by an S-shaped curve, an improvement beyond the objectives here.

The predictions of skin smoothness based on Equation 11 are identical with those calculated from Prall's five parameter empirical equation. This test is less stringent than those given above because Prall's test panels did not assess relative smoothness, but rather rank of smoothness: for five samples of skin, the smoothest was assigned the number 5; the second smoothest is called number 4; etc. Using the arguments in this paper, we cannot only duplicate Prall's predictions, but also approximately predict the experimental data on which his predictions are based (Cussler, 1977).

In the analysis above, we assumed that subjective thinness was closely related to the reciprocal of the viscous force on the fingers. The data which permit the test of this assumption are given in Table 4 . The first two columns give the rheological properties of the fluids; the third gives the velocities measured in 
the assessment; and the fourth column gives the viscous force per area calculated from Equation 14.

Figure 5 shows that the perceived thinness is, in fact, closely related to this viscous force. The values on the ordinate again represent psychophysical assessments; the data on the abscissa are viscous forces per area, found from nonlinear combinations of purely physical parameters. The correlation between the subjected assessments and the calculated forces is -0.91 , but this correlation again rests strongly on outlying points. While this is not as high as previous correlations, the liquids used here show a much wider range of non-Newtonian behavior. This wider range means that the assumption of steady motion made in deriving Equation 14 is much more critical.

\section{DISCUSSION}

The results above show that tactile assessments can be successfully correlated with nonlinear combinations of physical parameters. These combinations are predicted from physical models of the forces on the skin's surface. More specifically, smoothness is associated with the friction force, which is, in turn, a known function of grit diameter, coefficient of friction, and Young's modulus. Thinness is related to the viscous force, which in turn is a known function of the various physical parameters describing non-Newtonian fluids.

These results provide a scheme for generalizing

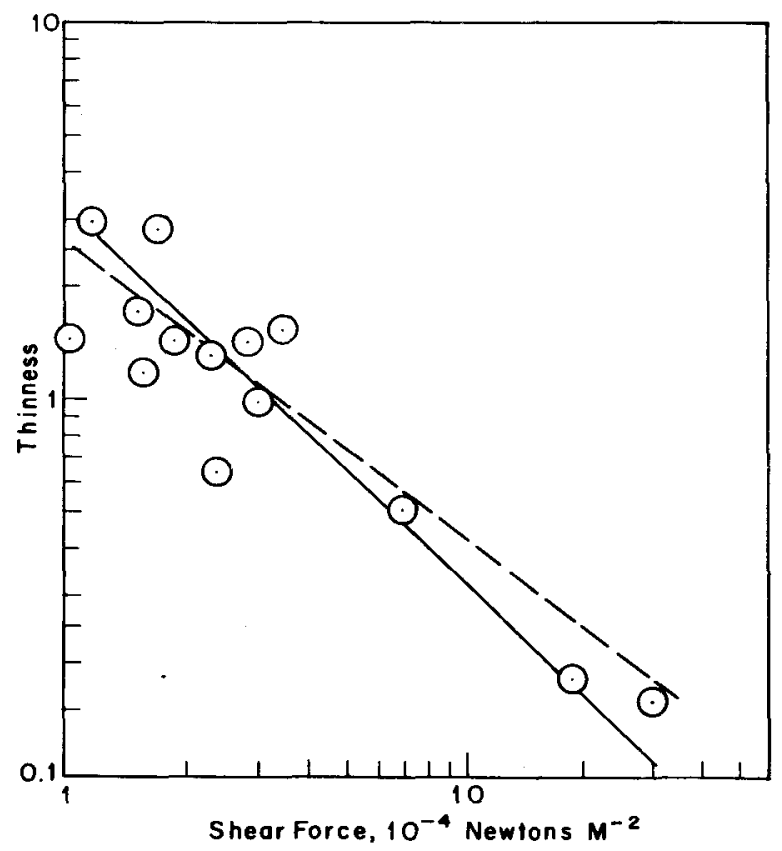

Figure 5. Thinness vs. shear force. The subjective values are shown on the ordinate; the viscous shear forces on the abscissa are found from the rheological data in Table 4 . The solid line of slope -1 is that expected from Equation 10; the dotted line of slope -0.81 is the best fit.

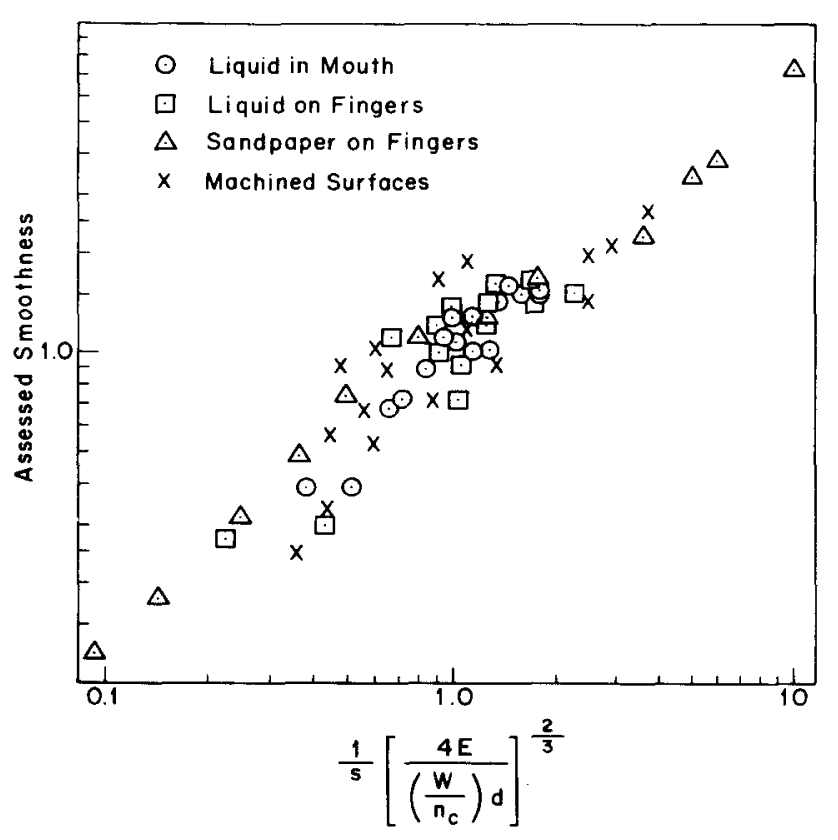

Figure 6. Smoothness in different situations. The psychophysically assessed smoothness is plotted vs. the reciprocal of the friction force for four different types of stimulus. For each type, the friction force involves variation of different physical properties.

empirical correlations like Stevens' law. To illustrate this scheme, the subjective assessments of four different studies related to smoothness are superimposed and plotted vs. the reciprocal of the friction forces in Figure 6. Because the different studies used different panels and different stimuli, the absolute values of smoothness cannot be directly compared between studies. The important point is that each study involves systematic variation of different physical parameters. The assessments of smoothness on the fingers, plotted from Table 3 as squares, change most with shear strength, s, and with load, $\mathrm{W}$. The assessments in the mouth (Kokini, Kadane, \& Cussler, 1977), shown as circles, vary most with changes in s. The assessments of sandpaper (Stevens \& Harris, 1962), shown as triangles, are altered largely by changes in $d$. Finally, the assessments of machined surfaces (Lederman, 1974), plotted as crosses, involved a systematic variation only of the load W. This last data set are actually based on assessments of roughness, which we have assumed to be inversely proportional to smoothness (Stevens \& Harris, 1962).

All of the data fall along the same line. Thus the results are a partial approximation of a "general theory of texture psychophysics," in which new, previously unsuspected, combinations of physical variables are used in mapping of subjective responses to physical variables (Moskowitz, Drake, \& Akesson, 1972; Moskowitz \& Kapsalis, 1974). Similar arguments should provide guides in other situations. 
These conclusions depend on the accuracy of the correlations in Figures 4-6. To test this accuracy, we deliberately introduced both random and systematic errors in each of the physical factors in Equations 7 and 14, and then recalculated the data shown in Figures 4-6. More specifically, the load, the finger velocity, and the coefficient of friction were changed $30 \%$; and $\mathrm{m}$ and $\mathrm{n}$ were changed $10 \%$. The slopes of the best lines in these figures were commonly changed about 0.1 , especially by systematic errors in the normal force (in Figure 4) and in the finger velocity (in Figure 5). However, the correlation coefficients between the tactile assessments and the calculated forces commonly changed about 0.02 . Nevertheless, one should remember that these small changes in correlation depend strongly on the consistency of the outlying data points. The sensitivity over a smaller range of assessments would be greater.

These conclusions are largely consistent with earlier studies, but not completely so. The association of smoothness with friction force is in agreement with other measurements of the coefficient of friction on the fingers (Ekman, Hosman, \& Lindström, 1965) and in the mouth (Kokini et al., 1977). Earlier studies also seem to show that the smoothness of solid surfaces is inversely proportional to roughness (Stevens \& Harris, 1962). If this is so, Equation 10 can be inverted to give

$$
\text { roughness } \propto \mathrm{sW}^{2 / 3} \mathrm{~d}^{2} .
$$

This is roughly equal to the square root of the target function of $\mathrm{Wd}^{4}$ found empirically (Lederman \& Taylor, 1972; Lederman, 1974; Taylor \& Lederman, 1975). Because we are using logarithmic comparisons, these two combinations of physical parameters give very similar results. However, the variation of smoothness with the coefficient of friction shown in Figures 4 and 6 is not consistent with the independence of roughness and coefficient of friction reported elsewhere (Taylor \& Lederman, 1975). We are not sure why this is so. One possibility is that Taylor and Lederman did not measure the coefficient of friction as a function of both load and velocity (Moore, 1975) and then interpolate to the physical conditions acting on the skin.

The association of thinness with viscous force per area seems consistent with earlier measurements associating assessments of spreadability and viscosity with viscous force (deMartine \& Cussler, 1975; Kokini et al, 1977), which cannot only predict the Newtonian results of Stevens and Guirao (1964), but also provide estimates for non-Newtonian fluids. However, this work clearly does not explain visual assessments of Newtonian viscosity obtained by shaking a bottle (Stevens \& Guirao, 1964). It does explain tactile assessments of both smoothness and thinness.

The results in this work consequently provide a valuable guide for changing or duplicating tactile perceptions of commercial products. For example, one may increase smoothness by decreasing the size of bumps, by decreasing the shear strength of surface contacts, or by increasing Young's modulus of the skin. This last change is frequently accomplished by increasing skin hydration (Idson, 1973). In general, Equation 7 shows how physically different surfaces can have the same smoothness if they cause the same friction force.

One may manipulate thinness in a similar way. For equal thinness, fluids must engender an equal viscous force on the fingers. Two different power law fluids can easily produce the same force and hence have the same thinness. However, two Newtonian fluids of different viscosity will never have the same thinness. Such a means of duplication provides an interesting parallel with the "universal curves" reported for cosmetic consistency (Barry \& Grace, 1972; Barry $\&$ Meyer, 1973). These curves show that equal consistency requires specific values of shear stress and strain rate, values estimated in this work from fluid mechanics.

One example of this manipulation is provided by the thinness of fluids $D$ and $E$ reported in Table 4 . The viscous parameter $\mathbf{n}$ for fluid $\mathrm{E}$ is about 1 , so that fluid is almost Newtonian. In other words, from Equations 12-13, doubling the finger velocity doubles the viscous force. The parameter $n$ for fluid $\mathrm{D}$ is closer to 0.4 , so this fluid is non-Newtonian. In this case, doubling the finger velocity increases the force only about $30 \%$. However, these fluids are calculated to generate viscous forces within $\pm 15 \%$, and so are assessed as almost equally thin. This is true even though the two fluids have completely different physical properties.

Table 4

Values for Estimating Thinness*

\begin{tabular}{|c|c|c|c|c|c|}
\hline Fluid & $\mathrm{m}$ & $\mathrm{n}$ & $\begin{array}{l}\text { Finger } \\
\text { Velocity }\end{array}$ & $\begin{array}{l}\text { Viscous } \\
\text { Force per } \\
\text { Area** }\end{array}$ & Thinness ${ }^{\dagger} \dagger$ \\
\hline $\mathbf{A}$ & 7.3 & .29 & 4 & 3.1 & .99 \\
\hline B & 7.3 & .46 & 5 & 1.5 & 1.7 \\
\hline $\mathrm{C}$ & 4.7 & .44 & 6 & 1.1 & 1.5 \\
\hline D & 12.9 & .43 & 5 & 1.9 & 1.4 \\
\hline $\mathbf{E}$ & 1.4 & .95 & 2 & 3.9 & 1.5 \\
\hline $\mathbf{F}$ & .45 & 1.0 & 2 & 2.7 & 1.4 \\
\hline $\mathbf{G}$ & 7300 & 1.2 & .3 & 30. & .16 \\
\hline $\mathbf{H}$ & 56 & .31 & 4 & 2.4 & 1.3 \\
\hline I & 36 & .27 & 4 & 1.6 & 1.2 \\
\hline $\mathbf{J}$ & .010 & 1.0 & 6 & 1.2 & 3.0 \\
\hline $\mathbf{K}$ & 52 & .24 & 4 & 2.4 & .64 \\
\hline $\mathbf{L}$ & .021 & 1.0 & 6 & 1.8 & 2.8 \\
\hline $\mathbf{M}$ & 91 & 1.0 & 1 & 19. & .18 \\
\hline $\mathbf{N}$ & 17 & .39 & 2 & 7.1 & .51 \\
\hline
\end{tabular}

*Units: $m[=]$ newtons $\sec ^{n} m^{-2} ;$ velocity $\left[=110^{-2} \mathrm{~m} \mathrm{sec}^{-1} ;\right.$ and shear force $I=J 10^{-4}$ newtons $\mathrm{m}^{-2}$.

**This calculation used Equation 14 with the load $w=.3$ newtons.

fThese values are the average subjective assessments found in the companion paper. 
However, one can see at least two ways in which the physical factors considered in this paper are not accurate. First, in the analysis, we have assumed that the most important textural attributes depend on independent physical events: smoothness requires friction from skin contact, and thinness involves a viscous force without skin contact. One can imagine cases where these different events will not exist. For example, if we were to assess highly viscous fluids, we might not ever be able to get the skin contact normally important in evaluating smoothness. In this case, we may assess smoothness as a viscous force. If we were to assess fluids of very low viscosity, we might quickly get skin contact, and so assess thinness as a contact friction force. Thus smoothness and thinness may not always involve two different events.

A second way in which the factors considered here are not a complete description of perception concerns the idealized geometry used in the calculations. For example, for thinness, we assumed that the fingers were solid, infinite, flat plates moving steadily over each other. Clearly, they are not. The fingers are not infinite in size and are probably wet differently by the different fluids used in the research. Differently wetted area can cause different viscous forces. In addition, the fingers don't move steadily, but generally oscillate during tactile perceptions. As a result, the velocity gradient must also oscillate. For non-Newtonian fluids, changes in velocity gradient can have a highly nonlinear effect on the viscous force (cf. Equation 12). Including factors like these in our theory might well improve the correlation in Figure 5 .

\section{CONCLUSIONS}

The results above show how the vocabulary used to describe liquid texture can be reduced to three key attributes. Two of these attributes, smoothness and thinness, are closely related to specific forces on the skin's surface. These forces are calculated from idealized physical models. The calculations lead to correlations between assessments of attributes of specific combinations of physical variables, and thus represent a scheme for generalizing empirical correlations like Stevens' law.

\section{REFERENCE NOTE}

1. Breuer, M. M. Private communication to E. L. Cussler, 1976.

\section{REFERENCES}

ARChARD, J. F. Elastic deformation and the contact of surfaces. Nature, 1953, 172, 918-919.

BARRY, B. W., \& GRACE, A. J. Sensory testing of spreadability: Investigation of rheological conditions operative during application of topical preparations. Journal of Pharmaceutical Sciences, $1972,61,335-341$.
BARRY, B. W., \& MEYER, M. C. Sensory assessment of spreadability of hydrophilic topical preparations. Journal of Pharmaceutical Sciences, 1973, 62, 1349-1354.

BowDEN, F. B., \& TABOR, D. The friction and lubrication of solids, Oxford: Clarendon, 1964.

Cussler, E. L. Predicting skin texture. In M. M. Breuer (Ed.), Cosmetic science. London: Academic Press, 1977.

Demartine, M. L., \& Cussler, E. L. Predicting subjective spreadability, viscosity and stickiness. Journal of Pharmaceutical Sciences, 1975, 64, 976-982.

Ekman, G., Hosman, J., \& Lindström, B. Roughness, smoothness, and preference: $A$ study of quantitative relations in individual subjects. Journal of Experimental Psychology, 1965, 70, 18-26.

ForT, T. Adsorption and boundary friction of polymer surfaces. Journal of Physical Chemistry, 1962, 66, 1136-1143.

Fredrickson, A. G. Principles and applications of rheology. Englewood Cliffs: Prentice-Hall, 1965.

GoldBerger, A. S. Econometric theory. New York: Wiley, 1964. Pp. $60 \mathrm{ff}$.

Godenberg, R. L., \& DE LA Rosa, C. P. Correlation of skin feel of emollients to their chemical structure. Journal of the Society of Cosmetic Chemistry, 1971, 22, 635-654.

HERTz, H. Über die Berührung fester elastischer Körper. J. reine angew. Math., 1886, 92, 156-171.

Howorth, $\mathrm{C}$. The handling of suiting, lingerie and dress fabrics. Journal of the Textile Institute, 1964, 55, 251-260.

IDson, B. Water and the skin. Journal of the Society of Cosmetic Chemistry, 1973, 24, 197-212.

KokınI, J. L. Predicting liquid food texture from fluid dynamics and lubrication theory. Doctoral dissertation, Carnegie-Mellon University, 1976.

Kokani, J. L., Kadane, J. B., \& Cussler, E. L. Liquid texture perceived in the mouth. Journal of Texture Studies, 1977, in press.

Lederman, S. J. Tactile roughness of grooved surfaces: The touching process and effects of macro- and micro-surface structure. Perception \& Psychophysics, 1974, 16, 385-395.

Lederman, S. J., \& TAYLOR, M. M. Fingertip force, surface geometry, and the perception of roughness by active touch. Perception \& Psychophysics, 1972, 12, 401-408.

Middleman, S. The flow of high polymers. New York: Interscience, 1968.

Moore, D. F. Principles and applications of tribology. Oxford: Pergamon, 1975.

Moskowitz, H. R., Drake, B., \& Akesson, C. Psychophysical measures of texture. Journal of Texture Studies, 1972, 3, 135-145.

Moskowitz, H. R., \& Kapsalis, J. G. Towards a general theory of psychophysics. Proceedings of the IV International Congress of Food Science Technology, 1974, 2, 102-120.

PRALL, J. K. Instrumental evaluation of the effects of cosmetic products on skin surfaces with particular reference to smoothness. Journal of the Society of Cosmetic Chemistry, 1973, 24, 693-707.

Stevens, S. S., \& Guirao, M. Scaling of apparent viscosity. Science, 1964, 144, 1157-1158.

Stevens, S. S., \& Harris, J. R. The scaling of subjective roughness and smoothness. Journal of Experimental Psychology, $1962,64,489-494$.

TAYLoR, M. M., \& Lederman, S. J. Tactile roughness of grooved surfaces. A model and the effect of friction. Perception \& Psychophysics, 1975, 17, 23-26.

VAN WAZER, J. R. Viscosity and flow measurement. New York: Interscience, 1963.

(Received for publication Ju ne 8, 1976; revision accepted April 4, 1977.) 